\title{
Context Aware Modification on the Object Based Image Analysis
}

\author{
Fatemeh Tabib Mahmoudi • Farhad Samadzadegan • \\ Peter Reinartz
}

Received: 6 January 2014 / Accepted: 4 February 2015

(C) Indian Society of Remote Sensing 2015

\begin{abstract}
Spectral similarities, spatial adjacencies and complex relationships between various object types lead to the recognition difficulties and ambiguities in complex urban areas. Using a knowledge base containing contextual information together with higher levels of modifications in the object based image analysis may improve recognition results in such a situation. In this paper, contextual information composed of regions' internal and external context are utilized for object classification and modification based on the pre-defined segmented regions in very high resolution (VHR) remotely sensed imagery. In the proposed methodology, defining region's internal context for each of the segmented regions, object based image analysis (OBIA) is performed. Then, for the modification of the wrongly classified regions due to the internal context similarities, region's external context is defined based on the mutual dependencies between neighboring regions in the image. Evaluation of the capabilities of the proposed context aware object recognition methodology is performed on the WorldView-2 satellite imagery over Rio de Janeiro in Brazil together with a digital surface model. The obtained results represent that using various contextual information together with higher levels of modifications on the object regions, some of the object recognition difficulties due to the spectral similarities, spatial adjacencies and mistakes in generating the classification rules are decreased.
\end{abstract}

F. Tabib Mahmoudi $(\bowtie) \cdot$ F. Samadzadegan

Department of Geomatics, University of Tehran,

P.O Box: 11365-4563 Tehran, Iran

e-mail: fmahmoudi@ut.ac.ir

P. Reinartz

Department of Photogrammetry and Image Analysis, Remote Sensing Technology Institute, German Aerospace Centre (DLR), Oberpfaffenhofen 82234, Weßling, Germany
Keywords Object recognition · Modification · Contextual information $\cdot$ Internal context $\cdot$ External context $\cdot$ Mutual dependency

\section{Introduction}

One of the objectives of deploying the advanced commercial remote sensing sensors was to increase the visibility of urban objects, by reducing per-pixel spectral heterogeneity and thereby improving land cover identification. New sensors and VHR remotely sensed imagery may provide a wealth of information for automatic object recognition in urban areas in order to reduce time and labor intensive tasks, associated with field surveying or manual digitizing (Myint et al. 2011).

Because of the complex nature and diverse composition of land cover types found within the urban environment, the production of urban land cover maps from VHR imagery is a difficult task (Shackelford and Davis 2003). This situation potentially leads to the lower accuracy in pixel based image classification approaches those only use spectral characteristics of input remotely sensed data (Zhou and Troy 2008; Blaschke 2010; Myint et al. 2011; Meng et al. 2012; Shackelford and Davis 2003;). Therefore, the need for highly accurate and regularly updated geo-spatial information cannot be met only by the advancements of sensor technology.

Many researchers have investigated the potential of the object based image analysis approaches for dealing with very high resolution images and complexities in urban areas (Lewinski and Zaremski 2004; Jacquin et al. 2008; Zhou and Troy 2008; Blaschke 2010; Peets and Etzion 2010; Maclean and Congalton 2011; Myint et al. 2011). As it is depicted in the previous researches in the field of object based image analysis, the accuracy of object 
recognition results in complex urban areas directly depends on the segmentation and knowledge based classification processes (Ivits et al. 2005; Platt and Rapoza 2008; Zhou and Troy 2008; Myint et al. 2011; Laliberte et al. 2012).

Segmentation quality is one of the most crucial aspects in object based image analysis (Clinton et al. 2010; Marpu et al. 2010; Meinel and Neubert 2004). It is commonly believed that there is no universal segmentation method that can be successfully used for all types of land cover objects. Therefore, oversegmentation (when the real world object is segmented into smaller sub-objects) and under-segmentation (when the real world object or parts of it becomes a part of another object) may occur in the segmentation results (Marpu et al. 2010). Moreover, formalizing expert knowledge and encapsulating it into the rule sets is often time, labor and cost-intensive. Despite the research efforts in the field of OBIA, automatic recognition and reconstruction of objects in urban areas from VHR remotely sensed data is not yet solved and is highly affected by various factors such as spectral and spatial heterogeneity between different kinds of objects and unpredictable shapes of urban objects (Blaschke et al. 2008; Myint et al. 2011).

In this paper, higher levels of modifications together with the contextual information within several steps of object based classification are proposed to be utilized in order to improve object recognition results based on VHR remotely sensed data.

\section{Contextual Information}

Context can be defined as any information that is not directly produced by the appearance of an object. Appearance information, based on the visual cues, can successfully identify object classes up to a certain extent. But, context that can be obtained from the nearby image data and neighboring objects, comprises any kind of relations between the semantic entities present in an image. Therefore, contextual information can help successfully disambiguate appearance inputs in the object recognition tasks (Wolf and Bileschi 2006; Galleguillos and Belongie 2010; Guo et al. 2013).

According to different levels of the utilized information, context can be further categorized concerning various points of view. In some researches, this categorization concerned several interaction levels; pixel interactions based on the notion that neighboring pixels tend to have similar labels, region interactions between image patches/segments and object interactions between various objects represent in the scene (Galleguillos and Belongie 2010; Guo et al. 2013).

Hermosilla et al., aim to define and analyze context-based descriptive features for classifying land-use in urban environments using three different object aggregation levels: objectbased, internal context, and external context. Object-based features composed of image-based, geometrical and threedimensional features. Internal context features describe an object with respect to the sub-objects contained within it. External context features characterize each object by considering the common properties of its adjacent objects (Hermosilla et al. 2012).

Guo et al., represent an object based classification procedure for high resolution images by exploiting different levels of contextual information. The contexts used in each stage were the object's inner context, the object's neighbor context and the object's scene context (i.e., the spatial homogeneity of the label distribution for different image objects and the consistency of feature distributions for different object classes in the whole scene), respectively. Their experimental results, which are based on a complex urban area, confirm that the neighbor context provides complementary information for the inner context and scene context (Guo et al. 2013).

\section{Proposed Object Recognition and Modification Methodology}

In this paper a context aware object recognition strategy composed of multiple steps is proposed for solving object recognition difficulties in complex urban areas, based on VHR remotely sensed imagery and digital surface model. As depicted in Fig. 1, the object recognition method is composed of two main stages: object based image analysis using region's internal context and object modification based on region's external context.

In the first stage, region's internal contextual information is utilized for object classification. Per segment spectral and textural pixels' interactions together with the structural features based on the size, shape and height of a segmented region, generate the internal contextual information for each region. This kind of contextual information is utilized for object based image analysis composed of image segmentation and object classification.

According to the spectral, textural and structural similarities between various kinds of objects in complex urban areas, initial object classification results may have some mistakes. Therefore, in the second stage of the proposed methodology, region's external contextual information based on the interactions between neighbouring regions is used for modifying the object classification results.

\section{Object Based Image Analysis}

Object based image analysis requires generating segmented regions as classification units. In this research, multiresolution segmentation technique is applied on the content of the VHR image in order to segment it into regions. The multi-resolution segmentation procedure starts with single image objects of one pixel and repeatedly merges a pair of image objects into larger ones. The merging decision is based on the local homogeneity criterion, describing the similarity between adjacent image objects (Baatz and Schape 2000). 


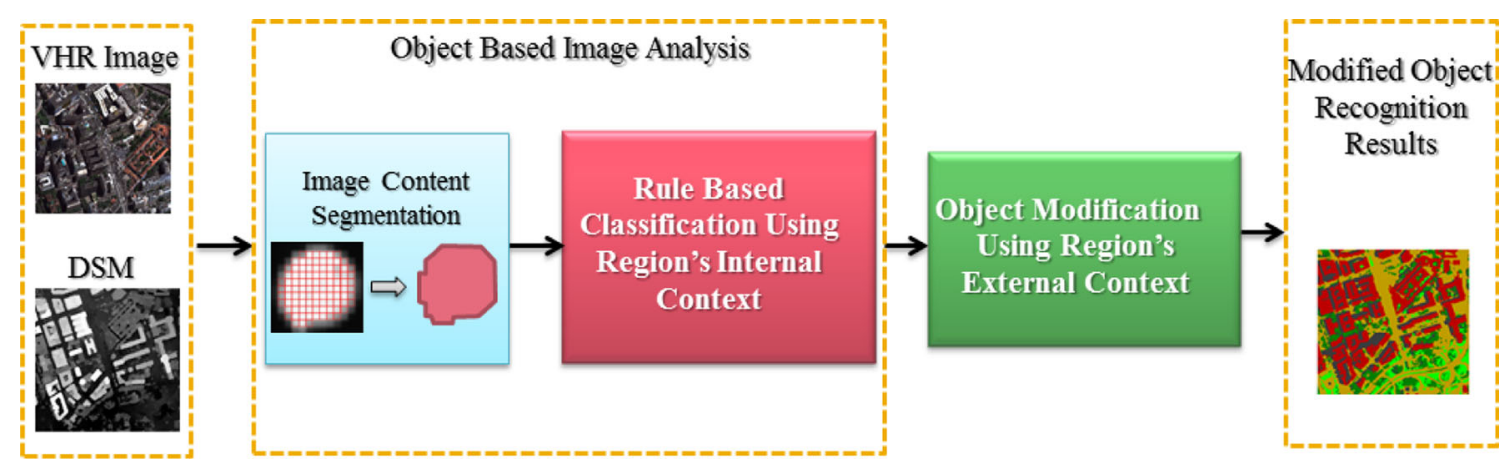

Fig. 1 General structure of the proposed object recognition strategy

After performing segmentation, a knowledge based classification process should be performed on each of the segmented regions. Therefore, it is necessary to gather proper knowledge composed of per-segment interactions between pixels and structural characteristics of each segmented region in order to provide internal context.

\section{Region's Internal Context}

The region's internal context as one of the main tools for performing knowledge based classification, is composed of spectral, textural and structural features:

- Spectral Features: The ratios between the reflectance values of various spectral bands are called index ratios; those can be used to establish the spectral interactions between pixels in a region. According to the spectral capabilities of the remotely sensed data, in this paper normalized difference indices (NDI) and simple ratios (SR) are used for proper definition of spectral features based on the image channels $\left(\right.$ band $\left._{1, k}\right)$.

$$
N D I_{k, l}=\left(\text { Band }_{k}-\text { Band }_{l}\right) /\left(\text { Band }_{k}+\text { Band }_{l}\right)
$$

$S R_{k, l}=$ Band $_{k} /$ Band $_{l}$

- Textural Features: Textural features can be measured based on the gray value relationships between each pixel and its neighbors in the pre-identified segmented regions. Some objects in urban areas such as buildings, road surfaces and grass lands have textural characteristics of the homogeneous regions and some other objects such as tree canopies have heterogeneous characteristics. Therefore, in this paper, we use energy, entropy, contrast and homogeneity in GLCM space together with the geo statistical semi-variogram features in order to measure textural features for various kinds of urban objects.

- Structural Features: Calculating suitable structural features based on the spatial characteristics and heights of the segmented regions provide another part of the internal context for using in the object classification process. In this paper, 2D structural features such as area, rectangularity, elongation and solidity are used for describing the shapes of various objects such as building, road surface and grass land. Moreover, relief and surface roughness are utilized as 3D structural features for discrimination between tree canopies and grass lands or buildings' roofs and road surfaces.

After generation of the above mentioned spectral, textural and structural features based on the image data and height products, an optimum selection of the features for recognizing individual object classes has the most effective role in generating a rich knowledge base of the region's internal context.

The object classification can be performed by encapsulating the knowledge base into a rule set and definition of a strategy for object recognition. The proposed strategy is a multi-process classification model that is a progressive process based on the spectral, textural and structural reasoning, respectively.

Despite the high potential of the strategies in the object based image analysis, wrongly classified regions due to 1) the similarities between internal context of various object types, 2) having no universally valid segmentation method for all urban object types and 3) complexities in generation of the rule set, decrease the reliability of the object recognition results. Therefore, performing the modification can improve the object classification accuracies.

\section{Modification Based on the Region's External Context}

Detecting the wrongly classified regions is the objective of performing modifications on the object classification results. Since, the proposed modification strategy is based on the region's external context; finding neighbouring regions is the main step of this process. Therefore, once the object based image analysis are performed, multiple operations should be done in order to use region's external context for detecting and modifying wrongly classified results (Fig. 2): 
- Step 1: Object categorization and coding is performed on the initial classification results in order to separate various object types.

- Step 2: Since, each of the codded object regions contains one or more sub-objects (segmented regions), it is necessary to distinguish individual sub-objects in each of the unique object codes in various classes.

- Step 3: Neighbourhood definition should be performed for each of the codded sub-objects in two ways:

- Neighbouring sub-objects having the same object code

- Neighbouring sub-objects with various object codes

For defining both kinds of the above mentioned neighbourhoods, it is assumed that the investigating region must locate on the border of its own containing object.

- Step 4: The mean values of the pre-defined features as region's internal context should be calculated for each of the codded sub-objects.

- Step 5: Calculating mutual information between each subobject and its pre-defined neighbours.

$$
\begin{aligned}
& \mathrm{MI}(\mathrm{x}, \mathrm{y})=\text { Entropy }_{(\mathrm{x})}+\text { Entropy }_{(\mathrm{y})}-\text { Joint Entropy }_{(\mathrm{x}, \mathrm{y})} \\
& \mathrm{x}=\left[\text { MeanFeature }_{\mathrm{il}}, \ldots, \text { MeanFeature }_{\mathrm{in}}\right] \\
& \mathrm{y}=\left[\text { MeanFeature }_{\mathrm{j} 1}, \ldots, \text { MeanFeature }_{\mathrm{jn}}\right]
\end{aligned}
$$

Where, $\mathrm{x}$ and $\mathrm{y}$ are the vectors containing mean feature values of two neighbouring sub-objects $i$ and $j$ and $\mathrm{MI}$ is the calculated mutual information between them.

- Step 6: Analysing all calculated mutual information for the codded sub-objects in order to detect and modify wrongly classified regions based on the following assumption, analysis and conclusion procedures:

- Assumptions

$$
\begin{aligned}
& \text { SubObject }_{\mathrm{i}} \in \quad \text { Object }_{\mathrm{M}} \\
& \text { Neighboured SubObject }{ }_{j} \in \text { Object }_{M}
\end{aligned}
$$

- Analysis

Condition $1_{(\mathrm{i}, \mathrm{M})} \rightarrow$ Low Dependency with Current Object $_{\mathrm{M}}$ Condition $2_{(\mathrm{i}, \mathrm{N})} \rightarrow$ High Dependency with Neighbour Object $_{\mathrm{N}}$

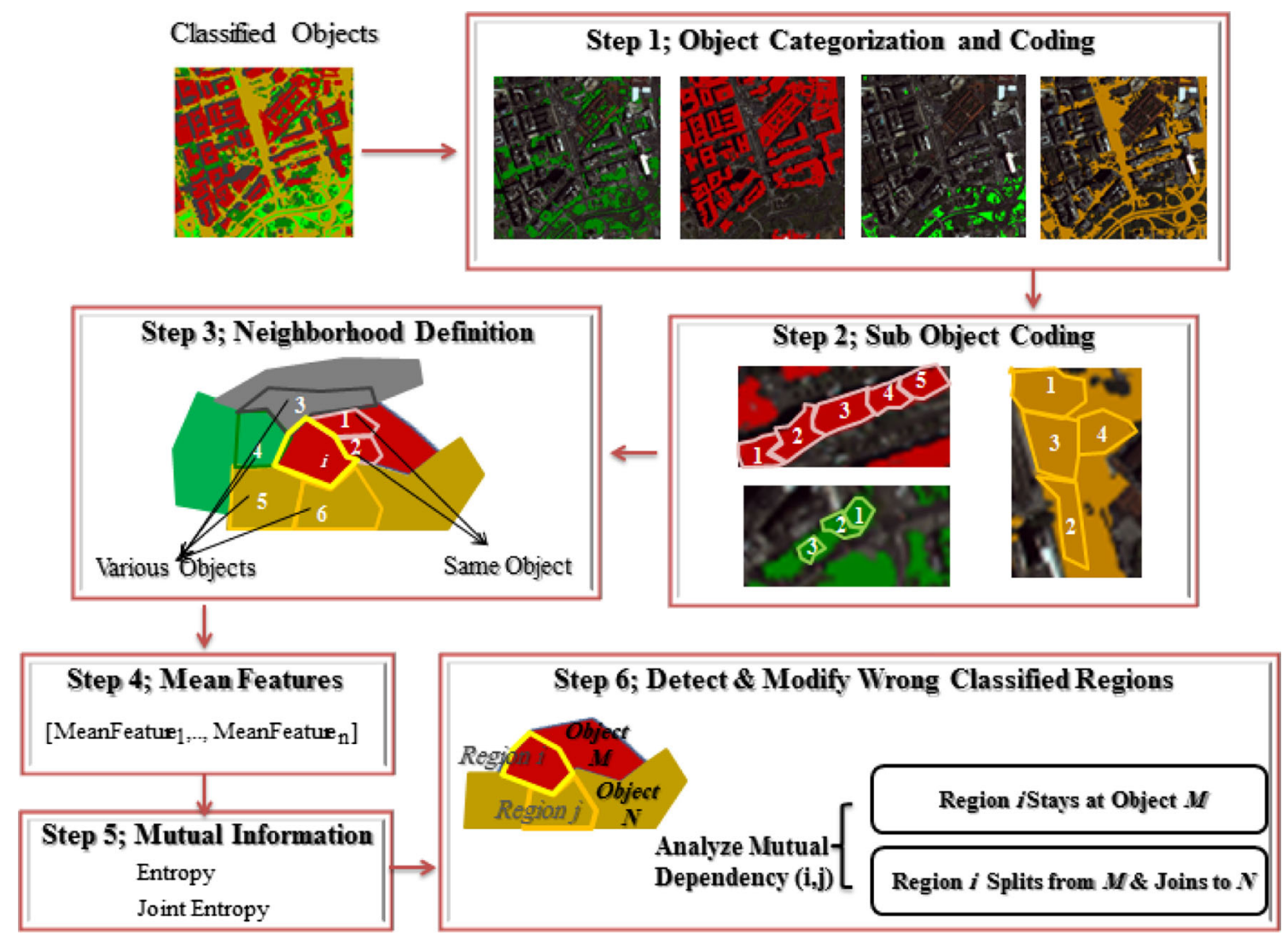

Fig. 2 Definition and Analysis of region's external context 
Condition $1_{(i, M)}$ : For neighbouring sub-objects having the same object code $M$, majority of small mutual information values for sub-object $i$ confirms independency with its neighbours in its current object.

Condition $2_{(i, N)}$ : For neighbouring sub-objects $i, j$ with different object codes $M \& N$, large amount of mutual information value for these two sub-objects confirms dependency between them.

- Conclusion If both conditions $1 \& 2$ are true, the subobject $i$ should be unclassified from its current object class $M$ and joint to its neighbour object class $N$ with highest dependency.

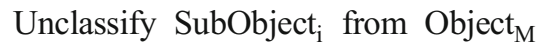
Joint SubObject ${\text { from } \text { Object }_{\mathrm{N}}}$

Concerning mutual dependencies between neighbouring regions as external context in modification decreases some of the difficulties related to the internal context similarities and mistakes in generating classification rules.

\section{Experiments and Results}

Dataset

The potential of the proposed object recognition and modification strategy is evaluated for automatic object recognition based on WorldView-2 satellite imagery over Rio de Janeiro (Brazil) which was collected in January 2010. Moreover, using multi-angular WorldView-2 images a digital surface model with a grid width of $50 \mathrm{~cm}$ is generated from all multiple pairs of panchromatic stereo images - in epipolar geometry - using the Semi-Global Matching (SGM) algorithm. This dataset contains the downtown area of the city, including the number of large and high buildings, commercial and industrial structures, and a mixture of community parks and private housing.

As depicted in Fig. 3, based on the variety of urban objects, we define two regions of interest (ROI $1 \&$ ROI 2) and five classes of objects in this study area. Building, road, tree, grass land and shadow area are the pre-identified object classes based on visual inspections. In the following sections, the outputs of each step of the proposed object recognition and modification methodology are represented for the sample patches, and finally, the results are shown for the whole area which is depicted in Fig. 3.

\section{Obtained Results}

In the stage of performing object based image analysis, the multi-resolution segmentation algorithm is applied to the content of VHR imagery using eCognition software version 8.7.
The values 90, 0.2 and 0.1 are used for the scale parameter, compactness and shape parameters, respectively. Then, various spectral, textural and structural features are measured on image regions for the generation of the knowledge base containing internal context and performing object level classification on the segmented regions. For generating spectral features all of the combinations between 8 spectral bands of WorldView- 2 image are used and only the panchromatic band of this image is utilized for measuring the textural features. For optimum selection of descriptors among all of the generated ones, it is necessary to perform diversity analysis. In this phase of our investigation, optimum features selection and threshold setting for each object class are performed semi-automatically by an expert operator using the quantitative and visual analysis on each of the features in feature view of eCognition software. Table 1 depicts the optimum selected features and their estimated thresholds for the generation of the rule set in the process tree tool of eCognition and performing object level classification on the segmented regions.

Within spectral classification, segmented regions are classified into five pre-defined object classes based on spectral knowledge and reasoning rules. Then, object level classification results are modified using textural knowledge and reasoning rules. Finally, using structural descriptors based on structural information of objects such as size, shape and height together with defining neighborhood relations between various kinds of object regions, the final candidate objects are obtained. In performing structural reasoning, the projection between image and ground (DSM) spaces are based on using the rational polynomial coefficients (RPC). Figure 4 illustrates the results of segmentation and multi-process object level classification on both selected sample patches. As it appears from obtained results, although it shows an acceptable potential for automatic object recognition, there are still some falsely recognized regions, especially in the adjacency of spectrally similar urban objects.

In the second stage of the object recognition methodology, the wrongly classified regions in recognition results are modified using region's external context based on the neighbourhood definition. According to the comparison between the results of the structural classification (last step of the object based image analysis) and the context aware modification (Fig. 4 (d1-d2) \& (e1-e2)), analysing mutual dependencies between neighbouring object regions decreases the classification mistakes due to the spectral and textural similarities in the classification rules. E. g. there is a gradual improvement in the classification of road region in the right and lower left areas of ROI 1 and upper left area of ROI 2. Moreover, Building region which wrongly classified as road are modified in the upper left area of the ROI 1.

For the quantitative evaluation of the results, some areas of the pre-defined object classes are manually selected on the image by an expert operator. The selected sample regions contain the total numbers of 159,250 pixels in the building 
Fig. 3 The dataset: a

WorldView-2 satellite image, b generated digital surface model from matching

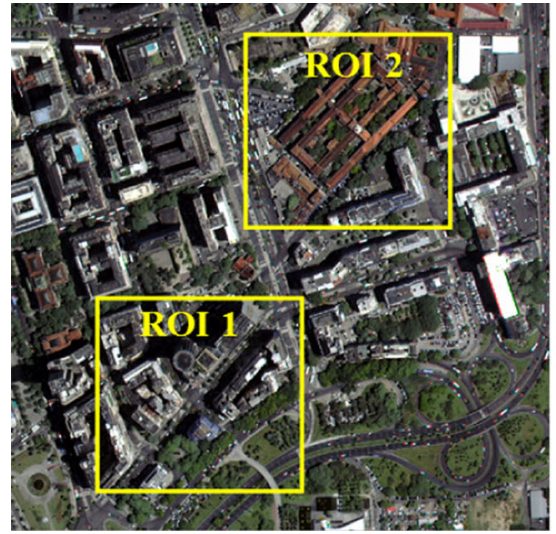

a

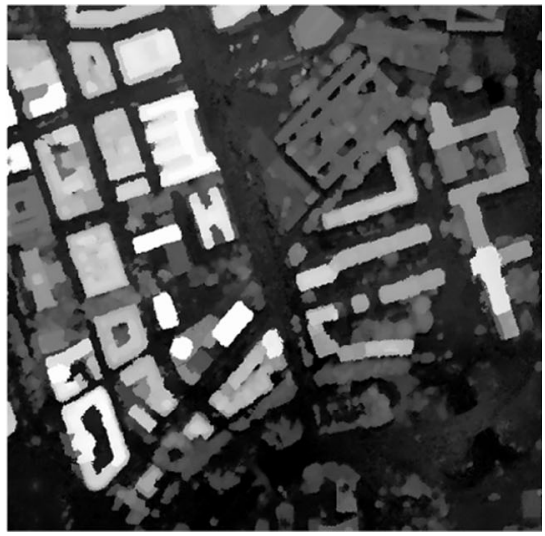

b class, 125,660 pixels in the road class, 56,979 pixels in the tree class, 10,666 pixels in the grass class and 101,580 pixels in the shadow class. Sample areas are compared with their corresponding results from different steps of the object recognition algorithm. As it is depicted in table 2, the comparison is based on the number of correctly detected pixels (true positive), wrongly detected pixels (false positive), and the not correctly recognized pixels (false negative), determined after the object recognition steps. The numbers of true positive, false positive and false negative pixels are evaluated based on the diagonal and non-diagonal elements of the generated confusion matrix for the obtained classification results. Moreover, using quantitative values for each object class, completeness, correctness and quality criteria are determined for the results (Congalton and Green 2009).

Completeness $=\frac{\text { TruePositive }}{\text { TruePositive }+ \text { FalseNegative }}$

Completeness $=\frac{\text { TruePositive }}{\text { TruePositive }+ \text { FalsePositive }}$
Quality $=\frac{\text { TruePositive }}{\text { TruePositive }+ \text { FalsePositive }+ \text { FalseNegative }}(9)$

Table 3 illustrates the considerable improvements of object recognition overall accuracies and Kappa through the object based image analysis process and context aware modification algorithm.

Figure 5 illustrates the gradual changes of completeness, correctness and quality factors for each of the pre-defined object classes in the multi-process object based image analysis and context aware modification.

\section{Discussions and Conclusion}

According to the difficulties in the field of automatic object recognition from very high resolution remotely sensed data, and because of the capabilities of the object based techniques and context aware systems, in this paper a context aware

Table 1 Optimum selected features and their thresholds

\begin{tabular}{|c|c|c|c|c|c|c|}
\hline \multirow[t]{2}{*}{ Optimum Features } & & \multicolumn{5}{|c|}{ Thresholds Based on Object Classes } \\
\hline & & Building & Road & Tree & Grass & Shadow \\
\hline \multirow[t]{5}{*}{ Spectral Features } & NDI(RE, CB) & $>-0.38 \&<-0.06$ & & & $>=-0.58 \&<=-0.3$ & \\
\hline & NDI(Red, RE) & $<0.4$ & & & & \\
\hline & SR(Green, Red) & & & $<1.3$ & & $<1.14$ \\
\hline & SR(NIR1, Yellow) & & & & & $>=1.6$ \\
\hline & SR(NIR2, RE) & & $>=1.25 \&<=1.45$ & $>2.8$ & & \\
\hline \multirow[t]{3}{*}{ Textural Features } & GLCM Homogeneity & $>0.45$ & $>=0.27 \&<=0.4$ & $<0.3$ & & $<0.2$ \\
\hline & GLCM Entropy & $>=4.8 \&<=5.5$ & $>=5.49 \&<=6$ & & & \\
\hline & GLCM Contrast & $>=50 \&<=190$ & $>=30 \&<=44$ & & & \\
\hline \multirow[t]{2}{*}{ Structural Features } & Rectangularity & $>0.4$ & $<=0.4$ & & & \\
\hline & Roundness & $<2$ & $>=2 \&<=4.5$ & $<2$ & $<2$ & \\
\hline
\end{tabular}




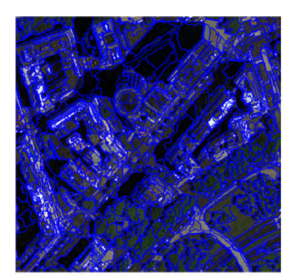

a1

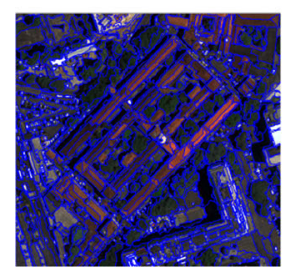

a2

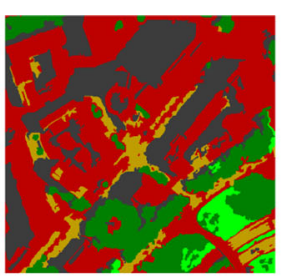

b1

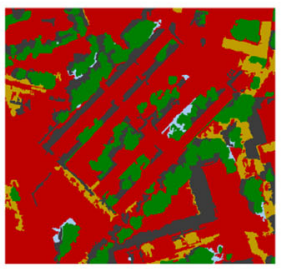

b2

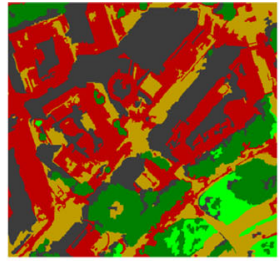

c1

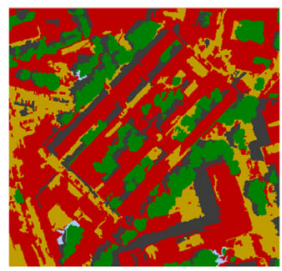

c2

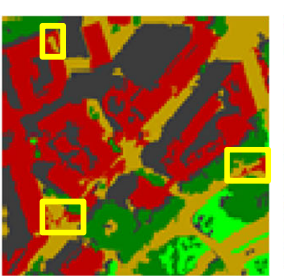

d1

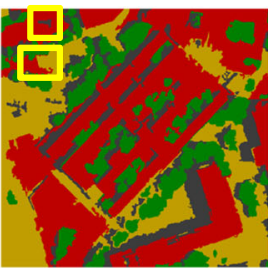

d2

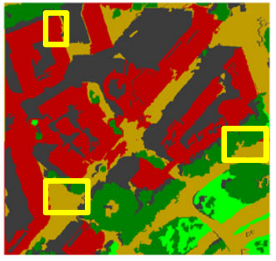

e1

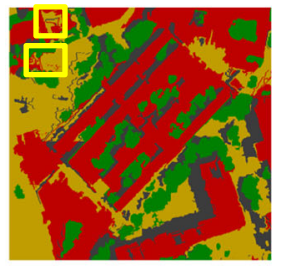

e2

Building Road Shadow Tree Grass

Fig. 4 Results of the object based image analysis and modification strategy on the first and second ROIs, respectively. a1-a2) Segmentation results, b1-b2) Spectral classification results, c1-c2) Textural

object recognition method is proposed for multiple object recognition based on WorldView-2 satellite imagery and digital surface model.

Spectral similarities between various object types in urban areas especially building and road or tree and grass, translate into some mistakes on the spectral classification results. This situation increases the number of false positive pixels of the object class building and decreases the number of true positive pixels of the road object class. Using textural features in the second step of the proposed object based image analysis classification results, d1-d2) Structural classification results. e1-e2) Results of the context aware modification on the object recognition results (Yellow boxes highlight improvements)

process can decrease the numbers of false positive pixels in the building class for about $14 \%$. On the other hand, textural similarities between various object classes also cause some other difficulties in the classification results. For instance, using textural information decreases the numbers of true positive pixels in the building class for about $13 \%$ and this reduces the completeness of the textural building classification results.

Using structural information based on sizes and shapes of objects together with topological relationships between them,

Table 2 Accuracy assessment of the object recognition results in both datasets

\begin{tabular}{|c|c|c|c|c|c|c|}
\hline \multirow[t]{2}{*}{ Recognition Step } & \multirow[t]{2}{*}{ Criteria } & \multicolumn{5}{|c|}{ Object Classes (\%) } \\
\hline & & Building & Road & Shadow & Tree & Grass \\
\hline \multirow[t]{3}{*}{ Spectral Classification } & True Positive & 97.2 & 21.1 & 88.7 & 92.7 & 71.5 \\
\hline & False Positive & 43.8 & 0.5 & 11.5 & 14.1 & 0.8 \\
\hline & False Negative & 7.3 & 78.9 & 11.3 & 7.3 & 28.4 \\
\hline \multirow[t]{3}{*}{ Textural Classification } & True Positive & 84.4 & 55.5 & 89.1 & 91.2 & 79.2 \\
\hline & False Positive & 30.0 & 12.6 & 11.9 & 13.2 & 1.2 \\
\hline & False Negative & 15.2 & 44.5 & 10.9 & 8.8 & 20.8 \\
\hline \multirow[t]{3}{*}{ Structural Classification } & True Positive & 94.1 & 76.8 & 89.2 & 91.2 & 79.2 \\
\hline & False Positive & 22.1 & 11.5 & 11.9 & 13.3 & 1.3 \\
\hline & False Negative & 5.9 & 23.2 & 10.8 & 8.8 & 20.8 \\
\hline \multirow[t]{3}{*}{ Modification Based on External Context } & True Positive & 94.7 & 78.6 & 84.8 & 91.3 & 79.2 \\
\hline & False Positive & 21.4 & 13.0 & 11.5 & 13.5 & 1.2 \\
\hline & False Negative & 6.3 & 21.4 & 15.2 & 8.7 & 20.8 \\
\hline
\end{tabular}


Table 3 Improvement in accuracies of multiple steps of the proposed object recognition methodology

\begin{tabular}{lllll}
\hline $\begin{array}{l}\text { Classification } \\
\text { Accuracies }\end{array}$ & \multicolumn{2}{l}{ Object Oriented Scene Analysis } & $\begin{array}{l}\text { Contextual } \\
\text { Modification }\end{array}$ \\
\cline { 2 - 5 } & $\begin{array}{l}\text { Spectral } \\
\text { Classification }\end{array}$ & $\begin{array}{l}\text { Textural } \\
\text { Classification }\end{array}$ & $\begin{array}{l}\text { Structural } \\
\text { Classification }\end{array}$ & \\
\hline Overall Accuracy (\%) & 68.33 & 82.0 & 86.97 & 89.63 \\
Kappa & 0.54 & 0.76 & 0.82 & 0.86 \\
\hline
\end{tabular}

increases the number of true positive pixels in both building and road classes. Due to the heterogeneity of the structural features, structural classification step doesn't have any significant effect on the results of tree, grass and shadow classes.
According to the results, from spectral to structural classification processes in the proposed object based image analysis, completeness, correctness and quality of the classification results especially in building and road classes are increased.
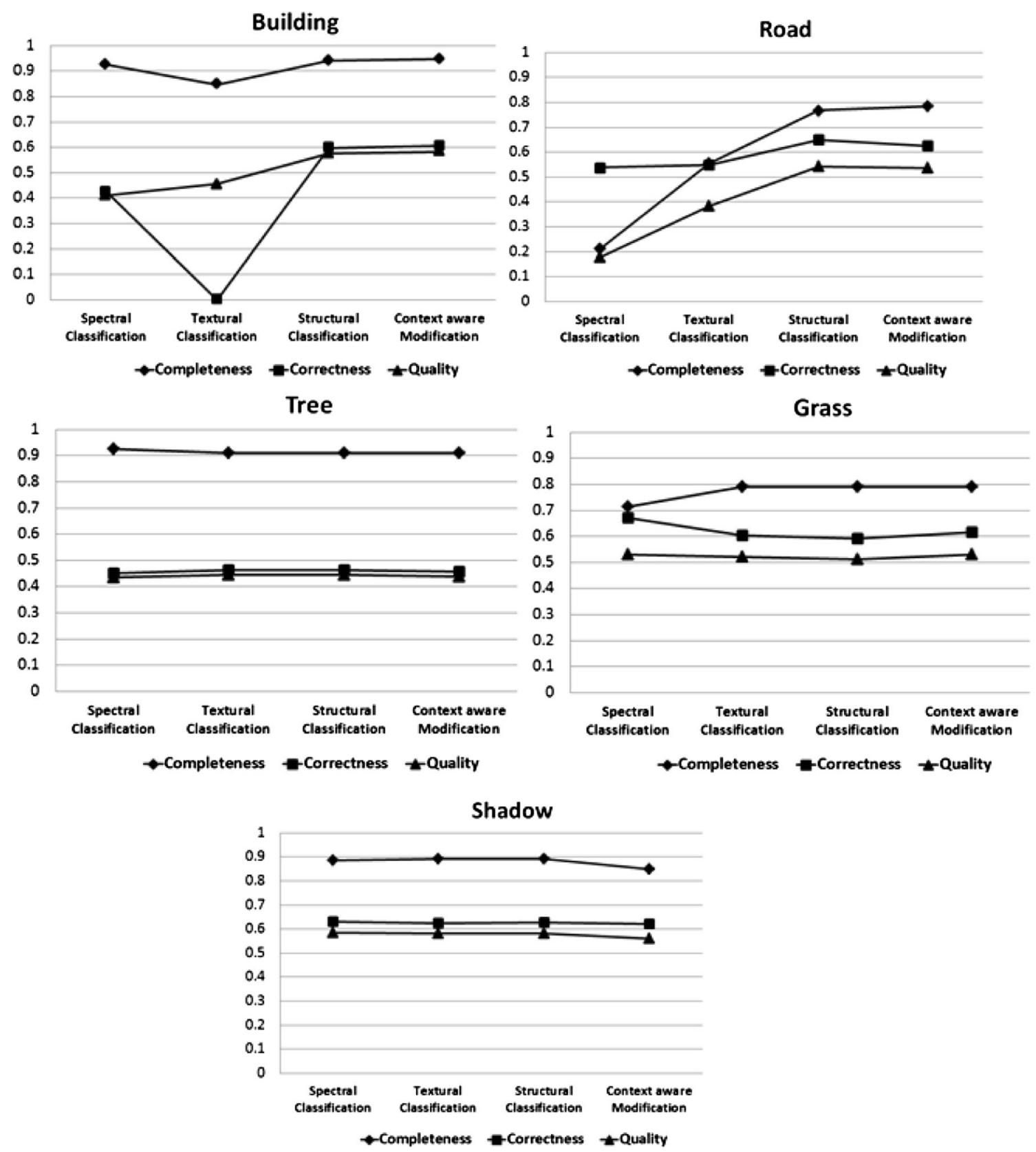

Fig. 5 Gradual changes in completeness, correctness and quality factors of multiple steps of the object recognition methodology 
Therefore, this object based image analysis method is so useful in the complex urban areas those have buildings with asphalt roofs. According to the results from the object based image analysis process, using the pre-defined spectral, textural and then structural features based on the region's internal context can improve overall accuracy and Kappa for about $18.64 \%$ and 0.32 , respectively.

On the other hand, complex nature of urban objects such as spectral similarity and spatial adjacency between various kinds of objects cause major difficulties in rule set definition and object recognition results based on the region's internal context. Neighbourhood definition together with calculating mutual dependencies between neighbouring object regions increase the number of true positive pixels in the classification results of some urban object types. As it is depicted in table 3, performing modification based on the region's external context improves overall accuracy and kappa values for about 2.66 and 0.04 , respectively.

However, this method still needs further modifications in the field of defining the contextual information used in various steps of the recognition process. Considering the global relationships between various object types throughout the scene, makes object recognition more reliable. Moreover, incorporating multi-angular or stereo images may be useful in order to solve object recognition difficulties due to shadow or occlusion in complex urban areas.

Acknowledgments The authors would like to thank IEEE GRSS data fusion technical committee for providing the dataset used in this paper.

\section{References}

Baatz, M., \& Schape, A. (2000). Multi-resolution segmentation: an optimization approach for high quality multi-scale image segmentation.In Angewandte Geographische Informationsverabeitung. XII. Beitragezum AGIT-Symp. Salzburg, T. Strobl, T. Blaschke and G. Griesebner (Eds), 12-23.

Blaschke T. (2010). Object based image analysis for remote sensing. ISPRS International Journal of Photogrammetry and Remote Sensing, 65(1), 2-16.

Blaschke T., Lang S., \& Hay G. J. (2008). "Object based image analysis: spatial concepts for knowledge-driven remote sensing applications, "lecture notes in geoinformation and cartography ISSN: 18632246. Springer.

Clinton, N., Holt, A., Scarborough, J., Li, Y., \& Peng, G. (2010). "Accuracy Assessment Measures for Object-based Image Segmentation Goodness." Photogrammetric Engineering \& Remote Sensing 76(3), $289-299$.
Congalton R. G., \& Green K. (2009). Assessing the Accuracy of Remotely Sensed Data: Principles and Practices, CRC Press, $137 \mathrm{p}$.

Galleguillos C., \& Belongie S. (2010). Context based object categorization: A critical survey. Image Understand: Comput. Vis.

Guo J., Zhou H., \& Zhu C. (2013). Cascaded classification of high resolution remote sensing images using multiple contexts. Information Sciences, 221(2013), 84-97.

Hermosilla T., Ruiz L. A., Recio J. A., \& Cambra-L pez M. (2012). Assessing contextual descriptive features for plot-based classification of urban Areas. Landscape and Urban Planning, 106(2012), 124-137.

Ivits E., Koch B., Blaschke T., Jochum M., \& Adler P. (2005). Landscape structure assessment with image grey-values and object-based classification at three spatial resolutions. International Journal of Remote Sensing, 26(14), 2975-2993.

Jacquin A., Misakova L., \& Gay M. (2008). A hybrid object-based classification approach for mapping urban sprawl in periurban environment. Landscape and Urban Planning, 84(2008), 152165.

Laliberte A. S., Browning D. M., \& Rango A. (2012). A comparison of three feature selection methods for object-based classification of sub-decimeter resolution UltraCam-L imagery. International Journal of Applied Earth Observation and Geoinformation, 15(2012), 70-78.

Lewinski, S., \& Zaremski, K. (2004). Examples of object oriented classification performed on high- resolution satellite images. WARSZAVA11, 349-358.

Marpu P. R., Neubert M., Herold H., \& Niemeyer I. (2010). Enhanced evaluation of image segmentation results. Journal of Spatial Science, 55(1), 55-68.

Meinel, G., \& Neubert, M. (2004). A comparison of segmentation programs for high resolution remote sensing data. Int. Arch. ISPRS35, 1097-1105.

Meng X., Currit N., Wang L., \& Yang X. (2012). Detect Residential Buildings from Lidar and Aerial Photographs through ObjectOriented Land-Use Classification (pp. 35-44). January: PE\&RS journal.

Myint S. W., Gober P., Brazel A., Grossman-Clarke S., \& Weng Q. (2011). Per-pixel vs. object-based classification of urban land cover extraction using high spatial resolution imagery. Remote Sensing of Environment, 115(5), 1145-1161.

Peets, A., \& Etzion, Y. (2010). Automated recognition of urban objects and their morphological attributes using GIS.ISPRS Archive XXXVIII (4-8-2-W9), "Core Spatial Databases- Updating, Maintenance and services-from theory to practice" Haifa, Israel.

Platt, R.V., \& Rapoza, L. (2008). An Evaluation of an Object-Oriented Paradigm for Land Use/Land Cover Classification. The Professional Geographer60(1), 87-100.

Shackelford A. K., \& Davis C. H. (2003). A Hierarchical Fuzzy Classification Approach for High-Resolution Multispectral Data over Urban Areas. IEEE Transaction on Geosciences and Remote Sensing, 41(9), 1920-1932.

Wolf L., \& Bileschi S. (2006). A critical view of context. International Journal of Computer Vision, 69(2), 251-261.

Zhou W., \& Troy A. (2008). An Object-Oriented Approach for Analyzing and Characterizing Urban Landscape at the Parcel Level. International Journal of Remote Sensing, 29, 3119-3135. 